\title{
The Effect of Torque Differences for All-Suture Anchor Fixation Strength: A Biomechanical Analysis
}

\author{
Lucca Lacheta, M.D., Jon Miles, M.S., Brenton Douglass, B.A., and \\ Peter Millett, M.D., M.Sc.
}

\begin{abstract}
Purpose: To investigate the biomechanical influence of differential loading of suture strands (torque) on the fixation strength of knotted and knotless all-suture anchors. Methods: The biomechanical strength of 48 all-suture anchors was evaluated for 4 conditions in polyurethane foam blocks: (1) 12 knotted all-suture anchors loaded proportionately, (2) 12 knotted all-suture anchors with 1 suture strand bearing 50\% of total force (partial torque), (3) 12 knotted all-suture anchors with 1 strand fixated and the other loaded (full torque), and (4) 12 knotless all-suture anchors with the loop kept open via a fixed rod. Force for $1 \mathrm{~mm}$ and $2 \mathrm{~mm}$ of displacement and ultimate failure load were assessed. Results: For $1 \mathrm{~mm}$ of displacement, groups 2, 3, and 4 showed significantly lower forces than group 1 (all $P<.001$ ), with no statistically significant difference between groups 2 and $3(P=.516)$; for $2 \mathrm{~mm}$ of displacement, all groups showed significantly lower forces than group $1(P<.001)$, which positively correlated with applied torque. No differences in the mean ultimate loads observed between testing groups 1,2 , and 4 were noted, with $93.3 \pm 3.8 \mathrm{~N}, 91.4 \pm 4.7 \mathrm{~N}$, and $92.6 \pm 5.6 \mathrm{~N}$, respectively; however, group 3 exhibited a significantly lower mean ultimate load $(62.3 \pm 1.7 \mathrm{~N})$ than all other groups $(P$ $<.001)$. Conclusions: The ultimate failure load of knotted and knotless all-suture anchor fixation was partially affected by loading differentials between strands in this validated foam block model. Differential loading of knotted all-suture anchor fixation presented greater initial displacement when compared with symmetrically loaded knotted all-suture anchors. Despite an initial increase in displacement, knotless all-suture anchors showed similar ultimate failure loads to knotted all-suture anchors with strands loaded equally. Clinical Relevance: The role of suture strand loading imbalance on anchor fixation is variable and should be considered during placement and fixation of the repair constructs in a clinical setting.
\end{abstract}

S

oft-tissue repair with all-suture anchors has grown in popularity because of the sutures' ability to preserve bone stock, improve postoperative imaging after surgery, and facilitate easier revision surgery if necessary. ${ }^{1-4}$ These all-suture anchors consist of a soft upper section and a stiff lower section that allow the anchor to increase in diameter when tension is applied, securing it to the cortical bone. Owing to the soft component of all-suture anchors, secure fixation in the intramedullary space must be provided by gentle, simultaneous pulling of both suture limbs to have the anchor seated against the cortex. ${ }^{1}$

From Steadman Philippon Research Institute, Vail, Colorado, U.S.A. (L.L., J.M., B.D., P.M.); Center for Musculoskeletal Surgery, Charitè Universitaetsmedizin Berlin, Berlin, Germany (L.L.); Department of Orthopaedic Sports Medicine, Technical University of Munich, Munich, Germany (L.L.); and The Steadman Clinic, Vail, Colorado, U.S.A. (P.M.).

The authors report the following potential conflicts of interest or sources of funding: L.L. reports nonfinancial support in the form of fellowship support from Arthrex. P.M. is a consultant for Arthrex, Medbridge, and Springer Publishing; receives royalties from Arthrex, Medbridge, and Springer Publishing; receives grants from Arthrex; owns stock in VuMedi; and reports that his institution receives funding from Arthrex, Smith $\theta$ Nephew. Siemens, and
Previous studies have addressed concerns about the fixation strength of all-suture anchors owing to their soft component and have shown that the ultimate failure load of all-suture anchors is similar to that of established solid suture anchors. ${ }^{5-8}$ However, all-suture anchors have been shown to have different biomechanical properties than solid suture anchors in terms of greater initial displacement owing to their soft component, achievement of higher ultimate loads to failure in high-density bone, and last, stronger pullout strength in the physiological traction direction of the capsulolabral complex in labral repair-or of the

Össur, outside the submitted work. Full ICMJE author disclosure forms are available for this article online, as supplementary material.

Received July 9, 2020; accepted December 24, 2020.

Address correspondence to Peter Millett, M.D., M.Sc., Steadman Philippon Research Institute, The Steadman Clinic, 181 W Meadow Dr, Ste 400, Vail, CO 81657,U.S.A.E-mail:drmillett@thesteadmanclinic.com

(C) 2021 THE AUTHORS. Published by Elsevier Inc. on behalf of the Arthroscopy Association of North America. This is an open access article under the CC BY-NC-ND license (http://creativecommons.org/licenses/by-nc-nd/4.0/). 2666-061X/20847

https://doi.org/10.1016/j.asmr.2020.12.008 
supraspinatus in rotator cuff repair-rather than in the knot-tying direction. ${ }^{2,9,10}$ Anchor failure during, for example, knot tying is a clinical observation that suggests that asymmetrical suture strand loads are applied while tying the knot for knotted all-suture anchors or securing the self-locking mechanism for knotless allsuture anchors, which may result in additional drilling or even bone loss, with the need to increase the diameter of drilling.

The purpose of this study was to investigate the biomechanical influence of differential loading of suture strands (torque) on the fixation strength of knotted and knotless all-suture anchors. It was hypothesized that increased imbalance of applied loads on all-suture anchors would result in decreased biomechanical stability.

\section{Methods}

\section{Experimental Design}

The biomechanical stabilities of 48 all-suture anchors were evaluated in 4 separate testing groups: Group 1 comprised 12 knotted anchors (1.7-mm FiberTak Suture Anchors; Arthrex, Naples, FL) equally loaded on both suture strands (no torque); group 2 consisted of 12 knotted anchors with the second strand bearing 50\% of the amount of force as the first strand (partial torque), group 3 comprised 12 knotted anchors with 1 suture fixated and the other suture loaded to failure (full torque), and group 4 consisted of 12 knotless anchors (1.8mm FiberTak Suture Anchors; Arthrex) with the suture loop kept open around a fixed rod representing the loop of suture around the glenoid labrum in the surgical setting (knotless). Institutional review board approval was not required for this study. The experimental design is illustrated in Fig 1.

All anchors were tested in $20-\mathrm{lb} / \mathrm{ft}^{3}\left(0.32-\mathrm{g} / \mathrm{cm}^{3}\right)$ polyurethane foam test blocks (Sawbones; Pacific Research Laboratories, Vashon, WA). These blocks were used because of their similar density to native glenoid and because they allow one to control for the variation in bone density of cadaveric specimens. ${ }^{11-14}$ The anchors were inserted into the bone block following the manufacturer's instructions by drilling a pilot hole with the associated drill and spear drill guide perpendicular to the block, ensuring no significant variation in the depth or angle of insertion. All anchors were placed in a grid pattern equidistant from each other such that no anchor was within $2 \mathrm{~cm}$ of another anchor or the edge of the bone block.

\section{Biomechanical Testing}

Bone blocks were rigidly clamped to the base of a dynamic tensile testing machine (Electropuls E10000; Instron, Norwood, MA). Sutures were secured via 10

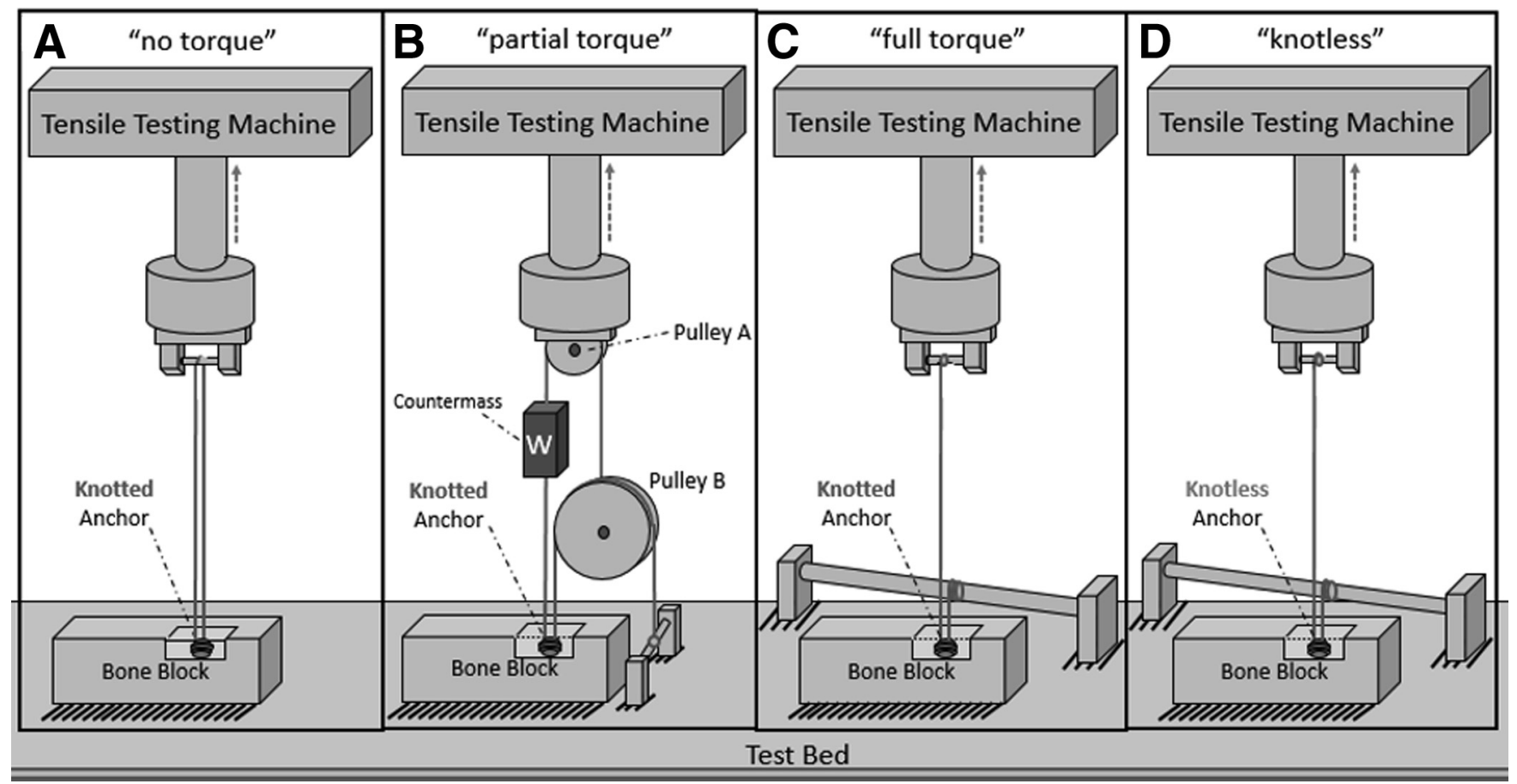

Fig 1. Testing apparatus for each testing group. (A) No-torque group. Both strands are tied together and equally tensioned around a bar on the actuator. (B) Partial-torque group. One strand bears $50 \%$ of the weight of the other strand. Pulley A has an outer diameter of 2 inches, pulley B has an outer diameter of 3.5 inches, and the counter mass and pulley B have equal weights of 1,245 g. (C) Full-torque group. One strand is secured to the bar on the actuator, and the other is secured to a rigid bar. (D) Knotless group. The repair suture is secured to the actuator of the dynamic tensile testing machine, and the loop is kept open around a rod on the surface of the bone block. Abbreviation: W, weight. 


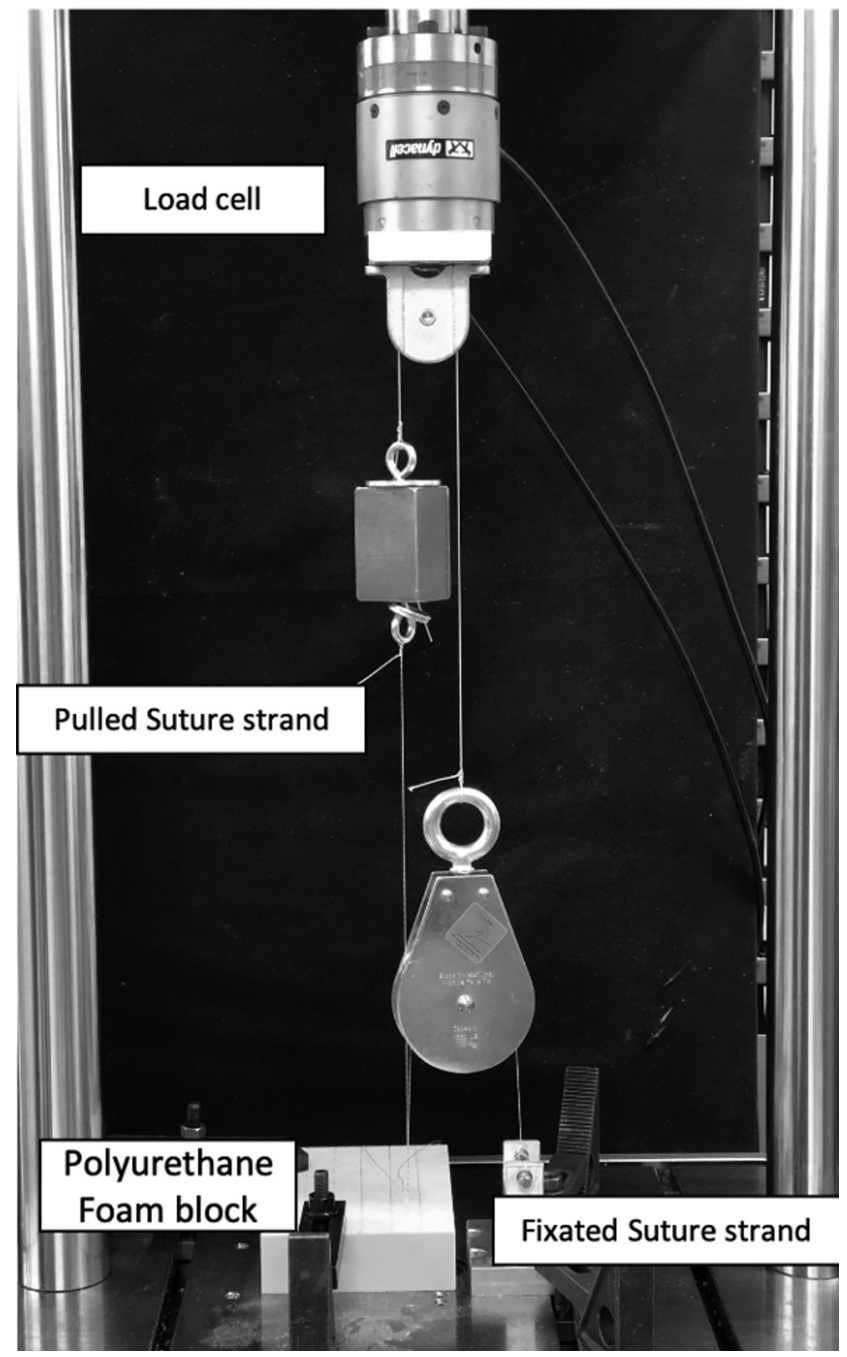

Fig 2. Biomechanical setup of $50 \%$ torque in group 2 .

knots to a custom apparatus designated for each testing group as shown in Fig 2. Care was taken to ensure that each strand of suture was vertically aligned during each trial, and the gap between the strands was minimized to the point where neither strand touched the other throughout testing.

Each trial consisted of a sequential preconditioning stage and a pull-to-failure stage. In the preconditioning stage, the anchors were cyclically tensioned from 5 to $50 \mathrm{~N}$ for 50 cycles at $0.5 \mathrm{~Hz}$. Afterward, the anchors were pulled to failure at a rate of $1 \mathrm{~mm} / \mathrm{s}$. Force and displacement data were recorded at $500 \mathrm{~Hz}$ throughout all trials. All raw data were processed programmatically using Matlab (version 2019a; The MathWorks, Natick, MA). To account for the potential error caused by coefficients of friction in the pulley in group 2, the forces experienced by the strands of the anchors were verified via load cell after data collection was completed to be within $\pm 1 \%$ of the expected forces in the range of failure $(75.0-112.5 \mathrm{~N})$ and within $\pm 1 \mathrm{~N}$ for all forces below the range of failure. Outcome measures reported in this article include the mean forces and standard deviations experienced by each anchor group at $1 \mathrm{~mm}$ and $2 \mathrm{~mm}$ of vertical displacement, as well as load to failure.

\section{Statistical Analysis}

Statistical analysis was performed using the statistical computing software R (2018 version; R Core Team/R Foundation for Statistical Computing, Vienna, Austria). A 1 -factor analysis of variance comparing the different testing groups was performed for forces at $1 \mathrm{~mm}$ of displacement and $2 \mathrm{~mm}$ of displacement, as well as load to failure. Statistically significant values were further refined using the Tukey HSD (honestly significant difference) test. The sample size of 12 anchors per group was determined based on a previous study with similar outcome parameters. ${ }^{15}$

\section{Results}

\section{Displacement Testing}

Within the knotted groups of anchors, with greater differentials of suture strand loading (higher torque), lower forces were required to displace $1 \mathrm{~mm}$. All group comparisons of force were significantly different (all $P$ $<.001)$ with the exception of group 2 versus group 3, which showed similar forces $(P=.516)$. Forces decreased from group 1 to group 2 and, finally, group 3, with those in group 4 (knotless anchors) lying between those in groups 1 and 2.

For tests with $2 \mathrm{~mm}$ of displacement, all group comparisons were significantly different, with groups 2 and 3 having the most similar forces required to displace 2 $\mathrm{mm}(P=.015$, with $P<.001$ for all other comparisons $)$. Specifically, group 1 exhibited the highest forces, followed by group 4 and then group 2; finally, group 3 showed the lowest forces.

\section{Load-to-Failure Testing}

No statistically significant differences in the mean ultimate loads observed during pull-to-failure testing were noted between groups 1 and $2(P=.683)$, between groups 1 and $4(P=.977)$, or between groups 2 and $4(P=.895)$; however, group 3 exhibited significantly lower mean ultimate loads than all other groups (all $P<.001$ ). The mean values with standard deviations for all 4 testing groups across all 3 tests are shown in Table 1, and their relative distribution is visually displayed in Fig 3. $P$ values for Tukey multiple comparisons of means are shown in Table 2.

\section{Discussion}

The main finding of this study was that the fixation strength of soft all-suture anchors was partially affected by differential loading (torque) of suture strands in a 
Table 1. Mean Forces at $1 \mathrm{~mm}$ and $2 \mathrm{~mm}$ of Displacement and Load to Failure Between Anchor Groups

\begin{tabular}{lccc}
\hline Group No. & Force at $1 \mathrm{~mm}, \mathrm{~N}$ & Force at $2 \mathrm{~mm}, \mathrm{~N}$ & Load to Failure, $\mathrm{N}$ \\
\hline 1 & $59.074 \pm 3.011$ & $71.559 \pm 2.313$ & $93.324 \pm 3.799$ \\
2 & $28.072 \pm 2.262$ & $49.887 \pm 3.709$ & $91.412 \pm 4.721$ \\
3 & $26.684 \pm 1.285$ & $45.959 \pm 3.079$ & $62.262 \pm 1.727$ \\
4 & $37.882 \pm 2.881$ & $61.121 \pm 2.755$ & $92.622 \pm 5.574$ \\
\hline
\end{tabular}

NOTE. Data are presented as mean \pm standard deviation.

foam block model. Furthermore, an increase in loading imbalance was positively correlated with displacement of all-suture anchors. Knotless all-suture anchors with unilateral suture strand loading while securing the selflocking mechanism were shown to exhibit similar ultimate failure loads to knotted all-suture anchors with both suture strands equally loaded (no torque).

This study aimed to quantify the effect of differential loading of suture strands on the forces sustained by allsuture anchors. It was hypothesized that higher torques would yield lower biomechanical stability; this was supported by significantly lower forces required to yield $1 \mathrm{~mm}$ and $2 \mathrm{~mm}$ of displacement in the disproportionately loaded groups 2, 3, and 4 when compared with testing group 1 with balanced loading across suture strands (all $P<.001$ ). Despite this variation in initial displacement, torque differences did not result in significant differences for ultimate failure loads, with $93.3 \pm 3.8 \mathrm{~N}, 91.4 \pm 4.7 \mathrm{~N}$, and $92.6 \pm 5.6 \mathrm{~N}$ for groups 1,2 , and 4 , respectively.

Group 1 served as a baseline for no or minimal torque in the clinical setting and yielded the highest forces required to displace $1 \mathrm{~mm}$ or $2 \mathrm{~mm}$, showing the highest biomechanical stability. On the contrary, group
3 showed the highest differential of loading across suture strands and, thereby, the lowest biomechanical stability. Likewise, group 2-with less torque application than group 3-showed no significant difference $(P=.683)$ in ultimate failure load when compared with group 1, which had balanced loading across strands; however, the force required to create initial displacement in group 2 ranged between that in group $1(P<$ $.001)$ and that in group $3(P=.015)$. Knotless all-suture anchors showed significantly lower displacement force $(P<.001)$ when compared with group 1 , without torque, but showed greater displacement force when compared with groups 2 and 3, with partial and full torque application, respectively (all $P<.001$ ). However, knotless all-suture anchors that generally generate torque during fixation were able to exhibit similar ultimate failure loads to knotted all-suture anchors tested without torque $(P=.977)$.

All-suture anchors have several characteristics in common. They are all based on a high-strength ultrahigh-molecular-weight polyethylene suture woven through a sleeve. ${ }^{16}$ The anchor is seated by gentle pulling of the suture to have the anchor seated against the cortex by folding the sleeve into a suture ball. This suture ball is larger than the cortical drill hole and prevents the anchors from pulling out.

The biomechanical strength of this soft-component fixation construct is influenced by several factors: Douglass et al. ${ }^{11}$ pointed out that higher-density bone is crucial for a strong all-suture anchor fixation. Barber and Herbert ${ }^{17}$ showed that the ultimate failure load of all-suture anchors is correlated directly with the number of sutures. Furthermore, the angle of insertion may be important, as shown by Oh et al. ${ }^{10}$ All-suture

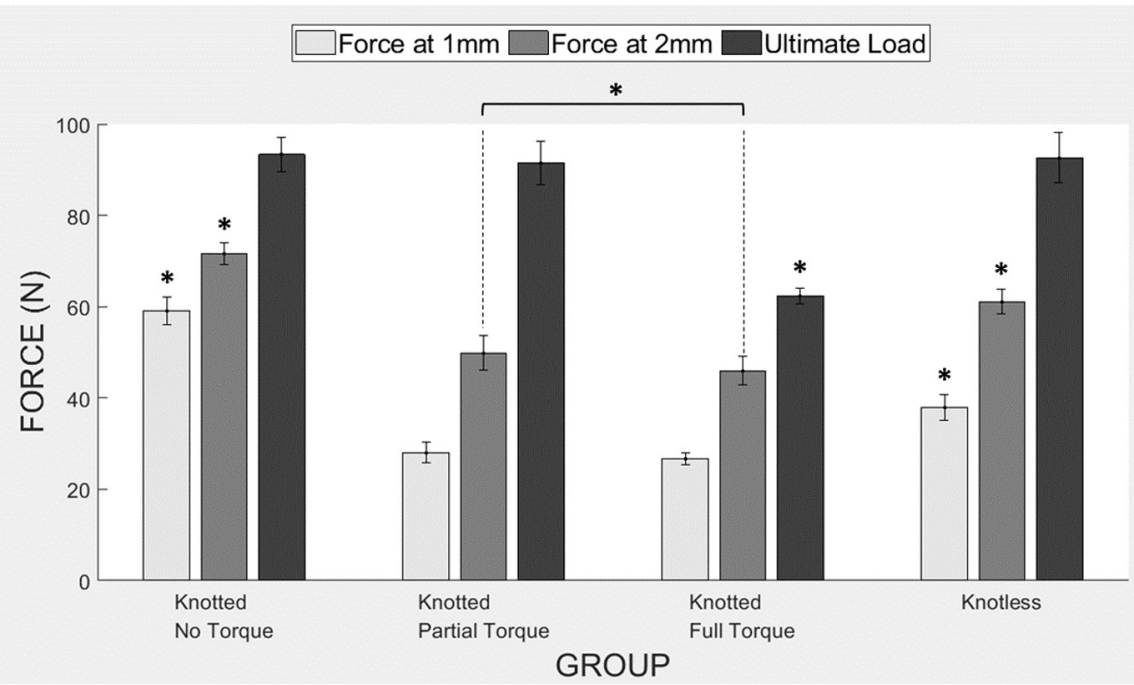

Fig 3. Bar plots with error bars (standard deviations) showing the force at $1 \mathrm{~mm}$ of displacement, force at $2 \mathrm{~mm}$ of displacement, and ultimate load in all testing groups. The asterisks above the bar plots denote statistically significant differences from all other groups. The asterisk with the bracket indicates a statistically significant difference for the direct comparison between 2 specific groups. 
Table 2. $P$ Values for Tukey Multiple Comparisons of Mean Results With $95 \%$ Family-wise Confidence Intervals

\begin{tabular}{|c|c|c|c|c|}
\hline & $\begin{array}{c}\text { Group 1: Knotted With } \\
\text { No Torque }\end{array}$ & $\begin{array}{l}\text { Group 2: Knotted With } \\
\text { Partial Torque }\end{array}$ & $\begin{array}{c}\text { Group 3: Knotted With } \\
\text { Full Torque }\end{array}$ & Group 4: Knotless \\
\hline \multicolumn{5}{|l|}{ Force at $1 \mathrm{~mm}, \mathrm{~N}$} \\
\hline Group 1: knotted with no torque & - & $<.001^{*}$ & $<.001^{*}$ & $<.001^{*}$ \\
\hline Group 3: knotted with full torque & $32.4[29.7$ to 35.1$]$ & $1.39[-1.29$ to 4.06$]$ & - & $<.001^{*}$ \\
\hline Group 4: knotless & $21.2[18.5$ to 23.9$]$ & $-9.81[-12.5$ to -7.13$]$ & $-11.2[-13.9$ to -8.52$]$ & - \\
\hline \multicolumn{5}{|l|}{ Force at $2 \mathrm{~mm}, \mathrm{~N}$} \\
\hline Group 3: knotted with full torque & $25.6[22.3$ to 28.9$]$ & $3.93[0.594$ to 7.26$]$ & - & $<.001^{*}$ \\
\hline Group 4: knotless & $10.4[7.17$ to 13.7$]$ & $-11.2[-14.6$ to -7.90$]$ & $-15.2[-18.4$ to -11.9$]$ & - \\
\hline \multicolumn{5}{|l|}{ Ultimate load, $\mathrm{N}$} \\
\hline Group 1: knotted with no torque & - & .6831 & $<.001^{*}$ & .9767 \\
\hline Group 2: knotted with partial torque & $1.91[2.67$ to 6.50$]$ & - & $<.001^{*}$ & .8946 \\
\hline Group 3: knotted with full torque & $31.1[26.5$ to 35.6$]$ & 29.1 [24.6 to 33.7$]$ & - & $<.001^{*}$ \\
\hline
\end{tabular}

NOTE. Data are presented as pair-wise group effects (Column - Row) in newtons [95\% confidence intervals] or as $P$ values.

*Statistically significant.

anchors presented stronger pullout strength in the physiological traction direction of the supraspinatus rather than in the knot-tying direction, consistent with the deadman theory. ${ }^{18}$ Stronger pullout strength was observed at the vertically directed insertion angle $\left(90^{\circ}\right)$, not at $45^{\circ} .^{10}$

Although $5 \mathrm{~mm}$ of displacement is considered clinically significant, for a labral or Bankart repair, $3 \mathrm{~mm}$ of gap formation or even less may be a concern. Ruder et al. ${ }^{19}$ have shown that 3 of 4 all-suture anchors tested exceeded the 5-mm limit during cyclic loading. When 3 mm was considered the cutoff, the number of failed allsuture anchors was even greater. This fact was mainly based on the soft component and tensioning technique of all-suture anchors compared with solid screw-in anchors. In a biomechanical comparison of ultimate load to failure and tensile displacement testing of allsuture anchors and screw-in anchors, Dwyer et al. ${ }^{9}$ concluded that hand-set anchors display low force displacement, which is likely because of slippage in the pilot hole. Pre-tensioned all-suture anchors were shown to eliminate this issue in their study.

The influence of differential loading of suture strands on the biomechanical performance of knotted and knotless all-suture anchor fixation had not been evaluated yet. This study's results suggest that strand loading imbalance during or after fixation of all-suture anchor constructs may influence biomechanical properties in terms of greater initial displacement and decreased ultimate failure load. Although displacement was very sensitive regarding varying levels of torque, ultimate failure loads of all-suture anchor fixation initially were not influenced by torque changes. It is interesting to note that knotless all-suture anchor fixation with a basic torque due to unilateral loading of the so-called repair suture ${ }^{1}$ during locking of the selflocking mechanism did not exhibit similar ultimate failure loads when compared with knotted all-suture anchor fixation without torque application; however, displacement of knotless all-suture anchors was found to be slightly lower when compared with the knotted anchors without torque. Regarding clinical implications, although all-suture anchors withstand a certain degree of torque in terms of ultimate failure load, differential loading of suture strands should be minimized during implantation because displacement forces are very sensitive and influenced by torque.

\section{Limitations}

This study is not without limitations. First, this was a biomechanical evaluation performed in polyurethane foam blocks and the results cannot be fully transferred to daily clinical practice; however, foam blocks allow one to control for variation in the bone density of cadaveric specimens. Second, only static biomechanical testing with cyclic pre-tensioning was performed. Testing protocols that use cyclic loading have been reported in the literature and may show different data. Third, this study solely focused on the biomechanical evaluation of all-suture anchors and was carried out in foam blocks that only reflect the density of human glenoid bone. Finally, as with all time-zero studies, healing properties cannot be evaluated, so caution should be taken when translating the observed results to an in vivo environment.

\section{Conclusions}

The ultimate failure load of knotted and knotless allsuture anchor fixation was partially affected by loading differentials between strands in this validated foam block model. Differential loading of knotted all-suture anchor fixation presented greater initial displacement when compared with symmetrically loaded knotted allsuture anchors. Despite an initial increase in 
displacement, knotless all-suture anchors showed similar ultimate failure loads to knotted all-suture anchors with strands loaded equally.

\section{References}

1. Lacheta L, Dekker TJ, Anderson N, Goldenberg B, Millett PJ. Arthroscopic knotless, tensionable all-suture anchor Bankart repair. Arthrosc Tech 2019;8:e647-e653.

2. Lacheta L, Brady A, Rosenberg SI, et al. Biomechanical evaluation of knotless and knotted all-suture anchor repair constructs in 4 Bankart repair configurations. Arthroscopy 2020;36:1523-1532.

3. Willemot L, Elfadalli R, Jaspars KC, et al. Radiological and clinical outcome of arthroscopic labral repair with allsuture anchors. Acta Orthop Belg 2016;82:174-178.

4. Lacheta L, Rosenberg SI, Brady AW, Dornan GJ, Millett PJ. Biomechanical comparison of subpectoral biceps tenodesis onlay techniques. Orthop J Sports Med 2019;7:232596711987627.

5. Ntalos D, Sellenschloh K, Huber G, et al. Conventional rotator cuff versus all-suture anchors-A biomechanical study focusing on the insertion angle in an unlimited cyclic model. PLoS One 2019;14:e0225648.

6. Goschka AM, Hafer JS, Reynolds KA, et al. Biomechanical comparison of traditional anchors to all-suture anchors in a double-row rotator cuff repair cadaver model. Clin Biomech 2015;30:808-813.

7. Otto A, Mehl J, Obopilwe E, et al. Biomechanical comparison of onlay distal biceps tendon repair: All-suture anchors versus titanium suture anchors. Am J Sports Med 2019;47:2478-2483.

8. Mazzocca AD, Chowaniec D, Cote MP, et al. Biomechanical evaluation of classic solid and novel all-soft suture anchors for glenoid labral repair. Arthroscopy 2012;28: 642-648.
9. Dwyer T, Willett TL, Dold AP, et al. Maximum load to failure and tensile displacement of an all-suture glenoid anchor compared with a screw-in glenoid anchor. Knee Surg Sports Traumatol Arthrosc 2016;24:357-364.

10. Oh JH, Jeong HJ, Yang SH, et al. Pullout strength of allsuture anchors: Effect of the insertion and traction angle-A biomechanical study. Arthroscopy 2018;34: 2784-2795.

11. Douglass NP, Behn AW, Safran MR. Cyclic and load to failure properties of all-suture anchors in synthetic acetabular and glenoid cancellous bone. Arthroscopy 2017;33:977-985.e5.

12. Kalouche I, Crépin J, Abdelmoumen S, Mitton D, Guillot G, Gagey O. Mechanical properties of glenoid cancellous bone. Clin Biomech 2010;25:292-298.

13. Mansat P, Barea C, Hobatho M-C, Darmana R, Mansat M. Anatomic variation of the mechanical properties of the glenoid. J Shoulder Elbow Surg 1998;7:109-115.

14. Frich LH, Jensen NC, Odgaard A, Pedersen CM, Søjbjerg JO, Dalstra M. Bone strength and material properties of the glenoid. J Shoulder Elbow Surg 1997;6:97-104.

15. Ergün S, Akgün U, Barber FA, Karahan M. The clinical and biomechanical performance of all-suture anchors: A systematic review. Arthroscopy 2020;2:e263-e275.

16. Barber FA. Editorial Commentary: All-suture shoulder glenoid anchors: Can we adequately tension them or knot? Arthroscopy 2019;35:1960-1963.

17. Barber FA, Herbert MA. All-suture anchors: Biomechanical analysis of pullout strength, displacement, and failure mode. Arthroscopy 2017;33:1113-1121.

18. Burkhart SS. Suture anchor insertion angle and the deadman theory. Arthroscopy 2009;25:1365 (letter).

19. Ruder JA, Dickinson EY, Peindl RD, Habet NA, Trofa DP, Fleischli JE. Cyclic and load-to-failure properties of allsuture anchors in human cadaveric shoulder glenoid bone. Arthroscopy 2019;35:1954-1959.e4. 